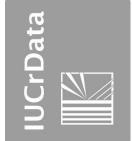

IUCrData

ISSN 2414-3146

Received 8 March 2018

Accepted 13 March 2018

Edited by W. T. A. Harrison, University of Aberdeen, Scotland

Keywords: crystal structure; redetermination; nickel; formate; hydrogen bonding.

CCDC reference: 1829415

Structural data: full structural data are available from iucrdata.iucr.org

\section{Redetermination of nickel(II) formate dihydrate}

\author{
Matthias Weil*
}

Institute for Chemical Technologies and Analytics, Division of Structural Chemistry, TU Wien, Getreidemarkt 9/164-SC, A-1060 Vienna, Austria. *Correspondence e-mail: matthias.weil@tuwien.ac.at

In comparison with the previous structure determination of poly[diaquadi- $\mu$ formato-nickel(II)], $\left[\mathrm{Ni}(\mathrm{HCOO})_{2}\left(\mathrm{H}_{2} \mathrm{O}\right)_{2}\right]_{n}$, based on Weissenberg film data [Krogmann \& Mattes (1963). Z. Kristallogr. 118, 291-302], the current redetermination from modern $\mathrm{CCD}$ data revealed the positions of the $\mathrm{H}$ atoms, thus making a detailed description of the hydrogen-bonding pattern possible. Both $\mathrm{Ni}^{2+}$ cations in the crystal structure are located on inversion centres and are octahedrally coordinated. One $\mathrm{Ni}^{2+}$ cation is bound to six $\mathrm{O}$ atoms of six formate anions whereas the other $\mathrm{Ni}^{2+}$ cation is bound to four $\mathrm{O}$ atoms of water molecules and to two formate $\mathrm{O}$ atoms. In this way, the formate anions bridge the two types of $\mathrm{Ni}^{2+}$ cations into a three-dimensional framework. $\mathrm{O}-\mathrm{H} \cdots \mathrm{O}$ hydrogen bonds of medium strength between water molecules and formate $\mathrm{O}$ atoms consolidate the packing.
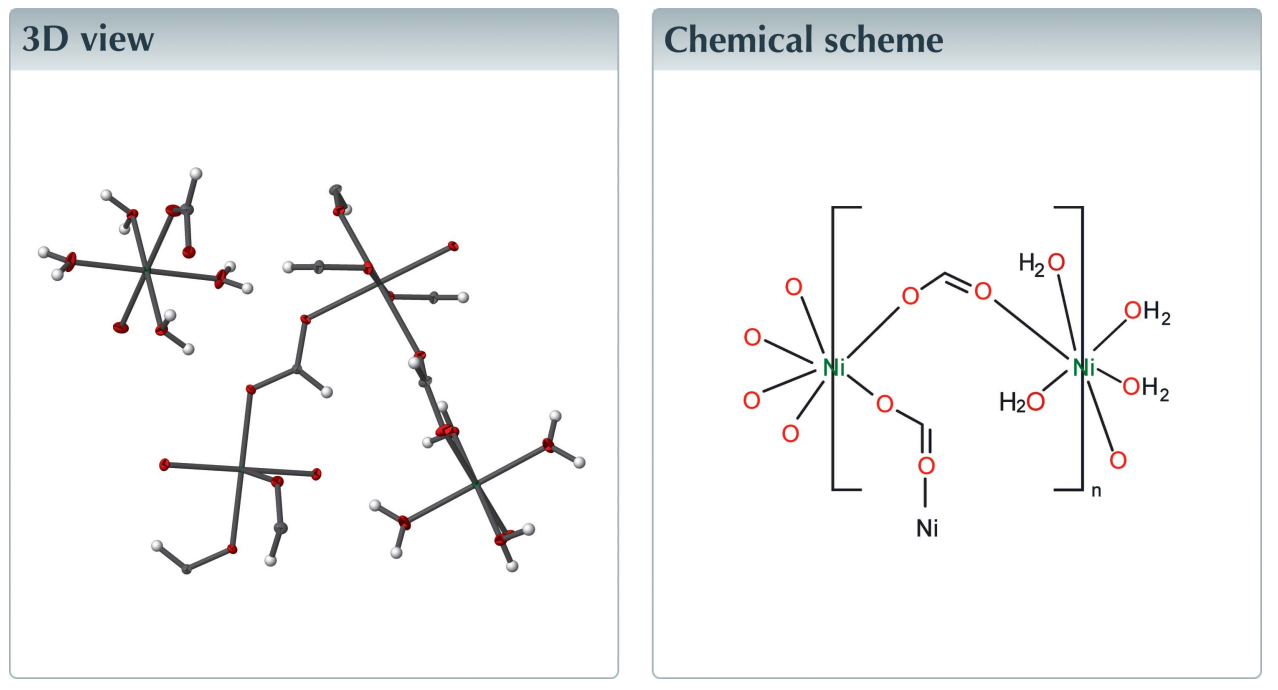

\section{Structure description}

Recycling of tungsten carbide from WC-Ni hard metals or composites thereof can be achieved by debinding WC-Ni with formic acid to selectively dissolve nickel. Nickel formate then can either be crystallized as the dihydrate from the obtained solution, or formic acid can be regenerated through cation exchange with sulfuric acid. In the latter case, nickel can be precipitated as $\mathrm{Ni}(\mathrm{OH})_{2}$ from the intermediate nickel sulfate solution by adding caustic soda (Weissensteiner, 2012). In the course of these studies it became apparent that a redetermination of the crystal structure of nickel formate dihydrate, $\mathrm{Ni}(\mathrm{HCOO})_{2} \cdot 2 \mathrm{H}_{2} \mathrm{O}$, (Krogmann \& Mattes, 1963) was desirable in terms of higher precision and accuracy and for an unambiguous assignment of the hydrogen-bonding scheme. Although a profile refinement using the Rietveld method has been performed on this material, leading to precise room-temperature lattice parameters (Kellerman et al., 2016), improved structural data are still missing. 
Table 1

Comparison of bond lengths $(\AA)$ in the current and the previous refinement of $\mathrm{Ni}(\mathrm{HCOO})_{2} \cdot 2 \mathrm{H}_{2} \mathrm{O}^{(a, b)}$.

\begin{tabular}{lll}
\hline & current refinement & previous refinement \\
\hline $\mathrm{Ni} 1-\mathrm{O}^{\mathrm{i}}$ & $2.0302(6)$ & $2.026(8)$ \\
$\mathrm{Ni} 1-\mathrm{O} 2$ & $2.0503(6)$ & $2.061(8)$ \\
$\mathrm{Ni1}-\mathrm{O} 3$ & $2.0942(6)$ & $2.097(8)$ \\
$\mathrm{Ni} 2-\mathrm{O} 5$ & $2.0256(7)$ & $2.042(8)$ \\
$\mathrm{N} 2-\mathrm{O} 6$ & $2.0663(6)$ & $2.059(8)$ \\
$\mathrm{Ni} 2-\mathrm{O} 4$ & $2.1006(7)$ & $2.090(8)$ \\
$\mathrm{O} 1-\mathrm{C} 1$ & $1.2593(10)$ & $1.256(8)$ \\
$\mathrm{O} 2-\mathrm{C} 1$ & $1.2546(10)$ & $1.222(8)$ \\
$\mathrm{O} 3-\mathrm{C} 2$ & $1.2618(10)$ & $1.278(8)$ \\
$\mathrm{O} 4-\mathrm{C} 2$ & $1.2607(10)$ & $1.247(8)$ \\
\hline
\end{tabular}

Symmetry codes: (i) $-x+1, y-\frac{1}{2},-z+\frac{1}{2}$; (ii) $x,-y+\frac{3}{2}, z+\frac{1}{2}$. Notes: (a) Krogmann \& Mattes (1963); lattice parameters $a=8.60(1), b=7.06$ (1), $c=9.21$ (2) $\AA, \beta=96.50(10)^{\circ}$ from single-crystal data at room temperature; (b) lattice parameters $a=8.5951$ (1), $b=$ $7.0688(5), c=9.2152(2) \AA, \beta=97.41$ (1) ${ }^{\circ}$ from Rietveld profile refinement at room temperature (Kellerman et al., 2016).

The crystal structure of $\mathrm{Ni}(\mathrm{HCOO})_{2} \cdot 2 \mathrm{H}_{2} \mathrm{O}$ comprises two $\mathrm{Ni}^{2+}$ cations on inversion centres, one on Wyckoff position $2 b$ (Ni1), one on $2 a(\mathrm{Ni} 2)$, and two formate anions and two water molecules in general positions. The $\mathrm{Ni}^{2+}$ cations are stacked in rows parallel to [101]. Both cations have a distorted octahedral coordination environment by oxygen atoms, but with different types of ligands. $\mathrm{Ni1}$ is bound to six $\mathrm{O}$ atoms of six formate

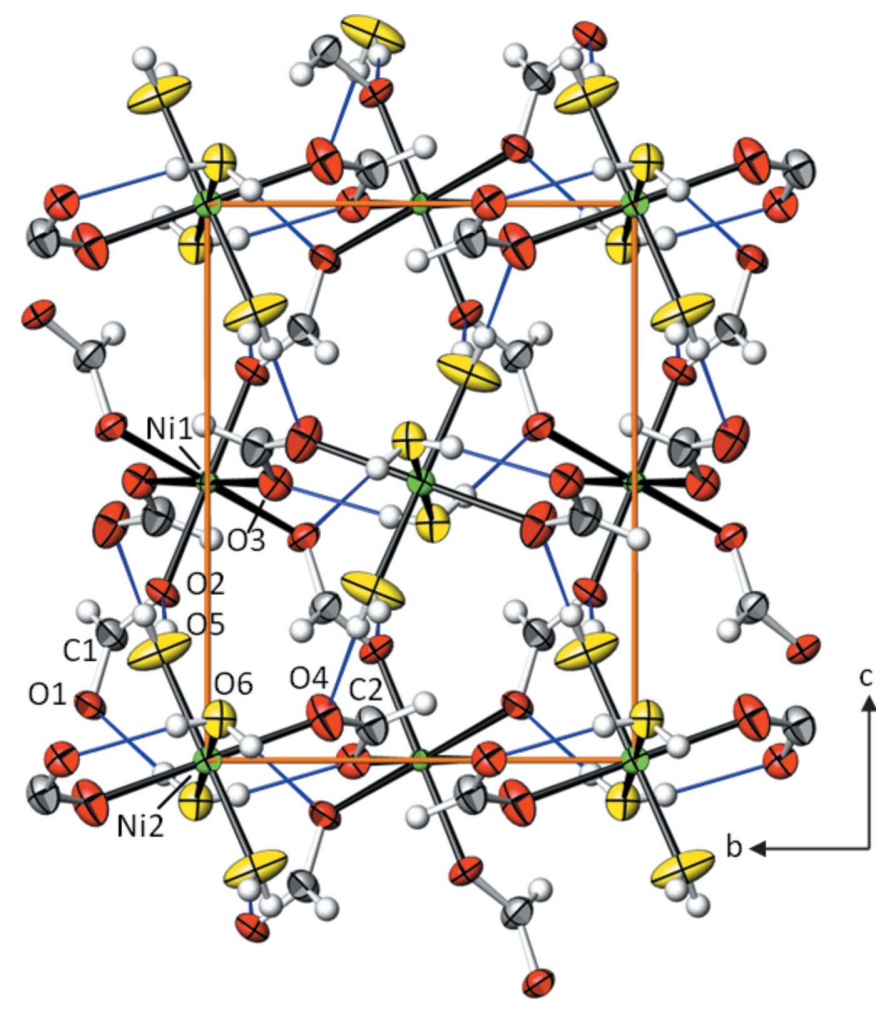

Figure 1

The crystal structure of $\mathrm{Ni}(\mathrm{HCOO})_{2} \cdot 2 \mathrm{H}_{2} \mathrm{O}$ in a projection along [100]. Displacement ellipsoids are drawn at the $97 \%$ probability level. $\mathrm{Ni}$ atoms are green, $\mathrm{C}$ atoms grey, formate $\mathrm{O}$ atoms red, water $\mathrm{O}$ atoms yellow. $\mathrm{H}$ atoms are shown as white spheres of arbitrary radius; $\mathrm{O}-\mathrm{H} \cdots \mathrm{O}$ hydrogen bonding is indicated by thin blue lines.
Table 2

Hydrogen-bond geometry $\left(\AA{ }^{\circ}{ }^{\circ}\right)$.

\begin{tabular}{|c|c|c|c|c|}
\hline$D-\mathrm{H} \cdots A$ & $D-\mathrm{H}$ & $\mathrm{H} \cdots A$ & $D \cdots A$ & $D-\mathrm{H} \cdots A$ \\
\hline $6-\mathrm{H} 5 \cdots \mathrm{O}^{\mathrm{i}}$ & $0.787(18)$ & $1.985(18)$ & $2.7312(9)$ & $158.1(16)$ \\
\hline $\mathrm{O} 5-\mathrm{H} 3$. & $0.89(2)$ & 1.87 & 2.7522 & $.2(19)$ \\
\hline $\mathrm{O} 5-\mathrm{H} 4 \cdots \mathrm{O} 4^{\mathrm{i}}$ & $0.832(18)$ & $1.898(18)$ & $2.7271(10)$ & 174.1 (17) \\
\hline $\mathrm{O} 6-\mathrm{H} 6 \cdots \mathrm{O} 1^{\mathrm{ii}}$ & $0.837(18)$ & $1.926(18)$ & $2.7610(9)$ & $175.5(16)$ \\
\hline
\end{tabular}

Symmetry codes: (i) $-x, y+\frac{1}{2},-z+\frac{1}{2}$; (ii) $-x,-y+2,-z$.

anions (O1-O3 and symmetry-related counterparts), whereas $\mathrm{Ni} 2$ is bound to four $\mathrm{O}$ atoms of two pairs of water molecules (O5, O6 and symmetry-related counterparts) and two formate anions (O4 and its symmetry-related counterpart). Relevant bond lengths and a comparison with the previous determination are collated in Table 1 . In general, bond lengths and angles are similar to related divalent first-row transition metal formates (Viertelhaus et al., 2005).

Each of the two formate anions bridges two $\mathrm{Ni}^{2+}$ cations, thus creating a three-dimensional framework. $\mathrm{O}-\mathrm{H} \cdots \mathrm{O}$ hydrogen bonds of medium strength and with nearly linear $\mathrm{O}-\mathrm{H} \cdots \mathrm{O}$ angles between water molecules as donor groups and each of the formate carboxylate $\mathrm{O}$ atoms as acceptor groups help to consolidate this arrangement (Fig. 1, Table 2). In comparison with the previous determination, the $\mathrm{H}$-atom positions are unambiguous and were clearly discernible from difference maps.

Table 3

Experimental details.

\begin{tabular}{|c|c|}
\hline Crystal data & \\
\hline Chemical formula & {$\left[\mathrm{Ni}(\mathrm{HCOO})_{2}\left(\mathrm{H}_{2} \mathrm{O}\right)_{2}\right]$} \\
\hline$M_{\mathrm{r}}$ & 184.78 \\
\hline Crystal system, space group & Monoclinic, $P 2_{1} / c$ \\
\hline Temperature $(\mathrm{K})$ & 100 \\
\hline$a, b, c(\AA)$ & $8.5806(4), 7.0202(3), 9.2257$ (4) \\
\hline$\beta\left(^{\circ}\right)$ & $97.551(1)$ \\
\hline$V\left(\AA^{3}\right)$ & $550.91(4)$ \\
\hline$Z$ & 4 \\
\hline Radiation type & Mo $K \alpha$ \\
\hline$\mu\left(\mathrm{mm}^{-1}\right)$ & 3.48 \\
\hline Crystal size $(\mathrm{mm})$ & $0.12 \times 0.10 \times 0.02$ \\
\hline Data collection & \\
\hline Diffractometer & Bruker APEXII CCD \\
\hline Absorption correction & $\begin{array}{l}\text { Multi-scan (SADABS; Bruker, } \\
\text { 2015) }\end{array}$ \\
\hline$T_{\min }, T_{\max }$ & $0.667,0.748$ \\
\hline $\begin{array}{l}\text { No. of measured, independent and } \\
\text { observed }[I>2 \sigma(I)] \text { reflections }\end{array}$ & $44072,3433,2591$ \\
\hline$R_{\text {int }}$ & 0.036 \\
\hline$(\sin \theta / \lambda)_{\max }\left(\AA^{-1}\right)$ & 0.907 \\
\hline Refinement & \\
\hline$R\left[F^{2}>2 \sigma\left(F^{2}\right)\right], w R\left(F^{2}\right), S$ & $0.021,0.049,1.04$ \\
\hline No. of reflections & 3433 \\
\hline No. of parameters & 101 \\
\hline $\mathrm{H}$-atom treatment & $\begin{array}{l}\mathrm{H} \text { atoms treated by a mixture of } \\
\text { independent and constrained } \\
\text { refinement }\end{array}$ \\
\hline$\Delta \rho_{\max }, \Delta \rho_{\min }\left(\mathrm{e} \AA^{-3}\right)$ & $0.52,-0.55$ \\
\hline
\end{tabular}

Computer programs: APEX3 and SAINT (Bruker, 2015), SHELXL2016 (Sheldrick, 2015), ATOMS (Dowty, 2006) and publCIF (Westrip, 2010). Coordinates from previous determination. 
Synthesis and crystallization

Crystals of the title compound were harvested from a saturated aqueous solution of nickel formate (Königswarter \& Ebell, Chemische Fabrik GmbH, Germany) that was stored in a closed glass bottle for several months.

\section{Refinement}

Crystal data, data collection and structure refinement details are summarized in Table 3. Starting coordinates for refinement were taken from the previous determination (Krogmann \& Mattes, 1963).

\section{Acknowledgements}

Dr Christian Weissensteiner kindly supplied the crystals used for this redetermination.

\section{Funding information}

The X-ray centre of TU Wien is acknowledged for financial support and for providing access to the single-crystal X-ray diffractometer.

\section{References}

Bruker (2015). APEX3, SAINT and SADABS. Bruker AXS Inc. Madison, Wisconsin, USA.

Dowty, E. (2006). ATOMS. Shape Software, Kingsport, Tennessee, USA.

Kellerman, D. G., Barykina, Yu. A., Zheleznikov, K. A., Tyutyunnik, A. P. \& Krasilnikov, V. N. (2016). Phys. Status Solidi B, 253, 2209 2216.

Krogmann, K. \& Mattes, R. (1963). Z. Kristallogr. 118, 291-302.

Sheldrick, G. M. (2015). Acta Cryst. C71, 3-8.

Viertelhaus, M., Adler, P., Clérac, R., Anson, C. E. \& Powell, A. (2005). Eur. J. Inorg. Chem. pp. 692-703.

Weissensteiner, C. (2012). Diploma thesis, TU Wien, Austria. Westrip, S. P. (2010). J. Appl. Cryst. 43, 920-925. 


\section{full crystallographic data}

IUCrData (2018). 3, x180428 [https://doi.org/10.1107/S2414314618004285]

\section{Redetermination of nickel(II) formate dihydrate}

\section{Matthias Weil}

Poly[diaquadi- $\mu$-formato-nickel(II)]

Crystal data

$\left[\mathrm{Ni}\left(\mathrm{CHO}_{2}\right)_{2}\left(\mathrm{H}_{2} \mathrm{O}\right)_{2}\right]$

$M_{r}=184.78$

Monoclinic, $P 2_{1} / c$

$a=8.5806$ (4) $\AA$

$b=7.0202(3) \AA$

$c=9.2257$ (4) $\AA$

$\beta=97.551(1)^{\circ}$

$V=550.91(4) \AA^{3}$

$Z=4$

$$
F(000)=376
$$

$D_{\mathrm{x}}=2.228 \mathrm{Mg} \mathrm{m}^{-3}$

Mo $K \alpha$ radiation, $\lambda=0.71073 \AA$

Cell parameters from 9891 reflections

$\theta=2.4-39.9^{\circ}$

$\mu=3.48 \mathrm{~mm}^{-1}$

$T=100 \mathrm{~K}$

Plate, green

$0.12 \times 0.10 \times 0.02 \mathrm{~mm}$

\section{Data collection}

Bruker APEXII CCD

3433 independent reflections

diffractometer

$\omega$ - and $\varphi$-scans

Absorption correction: multi-scan

(SADABS; Bruker, 2015)

$T_{\min }=0.667, T_{\max }=0.748$

44072 measured reflections 2591 reflections with $I>2 \sigma(I)$

$R_{\text {int }}=0.036$

$\theta_{\text {max }}=40.1^{\circ}, \theta_{\text {min }}=2.4^{\circ}$

$h=-15 \rightarrow 15$

$k=-12 \rightarrow 12$

$l=-16 \rightarrow 16$

\section{Refinement}

Refinement on $F^{2}$

Least-squares matrix: full

$R\left[F^{2}>2 \sigma\left(F^{2}\right)\right]=0.021$

$w R\left(F^{2}\right)=0.049$

$S=1.04$

3433 reflections

101 parameters

0 restraints

Hydrogen site location: mixed

$\mathrm{H}$ atoms treated by a mixture of independent and constrained refinement

$w=1 /\left[\sigma^{2}\left(F_{\mathrm{o}}^{2}\right)+(0.0181 P)^{2}+0.2503 P\right]$

where $P=\left(F_{\mathrm{o}}^{2}+2 F_{\mathrm{c}}{ }^{2}\right) / 3$

$(\Delta / \sigma)_{\max }=0.001$

$\Delta \rho_{\max }=0.52$ e $\AA^{-3}$

$\Delta \rho_{\min }=-0.55$ e $\AA^{-3}$

Special details

Geometry. All esds (except the esd in the dihedral angle between two 1.s. planes) are estimated using the full covariance matrix. The cell esds are taken into account individually in the estimation of esds in distances, angles and torsion angles; correlations between esds in cell parameters are only used when they are defined by crystal symmetry. An approximate (isotropic) treatment of cell esds is used for estimating esds involving l.s. planes.

Refinement. $\mathrm{H}$ atoms bound to $\mathrm{O}$ atoms were located from a difference map and were refined freely. 
Fractional atomic coordinates and isotropic or equivalent isotropic displacement parameters $\left(\hat{A}^{2}\right)$

\begin{tabular}{lllll}
\hline & $x$ & $y$ & $z$ & $U_{\text {iso }} / U_{\text {eq }}$ \\
\hline Ni1 & 0.500000 & 1.000000 & 0.500000 & $0.00423(3)$ \\
Ni2 & 0.000000 & 1.000000 & 0.000000 & $0.00564(3)$ \\
O1 & $0.40966(7)$ & $1.27481(9)$ & $0.09909(7)$ & $0.00780(10)$ \\
O2 & $0.40368(7)$ & $1.10493(9)$ & $0.30080(7)$ & $0.00783(10)$ \\
O3 & $0.29319(7)$ & $0.84112(9)$ & $0.49822(7)$ & $0.00847(10)$ \\
O4 & $0.06317(8)$ & $0.72530(10)$ & $0.07671(8)$ & $0.01179(11)$ \\
O5 & $0.08832(8)$ & $1.11264(12)$ & $0.19580(8)$ & $0.01638(14)$ \\
O6 & $-0.21606(8)$ & $0.97254(10)$ & $0.07381(7)$ & $0.00910(10)$ \\
C1 & $0.46712(10)$ & $1.22417(12)$ & $0.22586(9)$ & $0.00844(12)$ \\
H1 & 0.563988 & 1.279365 & 0.267077 & $0.010^{*}$ \\
C2 & $0.17660(10)$ & $0.61599(12)$ & $0.06198(10)$ & $0.00963(13)$ \\
H2 & 0.174105 & 0.491537 & 0.102054 & $0.012^{*}$ \\
H5 & $-0.2593(18)$ & $1.071(3)$ & $0.0623(17)$ & $0.023(4)^{*}$ \\
H3 & $0.188(2)$ & $1.098(3)$ & $0.2325(18)$ & $0.039(5)^{*}$ \\
H4 & $0.036(2)$ & $1.148(3)$ & $0.2609(19)$ & $0.033(5)^{*}$ \\
H6 & $-0.278(2)$ & $0.898(3)$ & $0.0248(18)$ & $0.031(4)^{*}$ \\
& & & &
\end{tabular}

Atomic displacement parameters $\left(\AA^{2}\right)$

\begin{tabular}{lllllll}
\hline & $U^{11}$ & $U^{22}$ & $U^{33}$ & $U^{12}$ & $U^{13}$ & $U^{23}$ \\
\hline Ni1 & $0.00443(5)$ & $0.00435(5)$ & $0.00395(5)$ & $-0.00014(4)$ & $0.00061(4)$ & $-0.00003(4)$ \\
Ni2 & $0.00466(5)$ & $0.00628(6)$ & $0.00601(6)$ & $0.00038(4)$ & $0.00083(4)$ & $-0.00025(5)$ \\
O1 & $0.0087(2)$ & $0.0080(2)$ & $0.0065(2)$ & $-0.00071(19)$ & $0.00013(18)$ & $0.00185(18)$ \\
O2 & $0.0081(2)$ & $0.0087(2)$ & $0.0066(2)$ & $-0.00015(19)$ & $0.00077(18)$ & $0.00260(19)$ \\
O3 & $0.0065(2)$ & $0.0094(2)$ & $0.0098(2)$ & $-0.00125(19)$ & $0.00243(18)$ & $-0.00016(19)$ \\
O4 & $0.0090(2)$ & $0.0106(3)$ & $0.0168(3)$ & $0.0033(2)$ & $0.0056(2)$ & $0.0040(2)$ \\
O5 & $0.0071(2)$ & $0.0307(4)$ & $0.0112(3)$ & $-0.0001(3)$ & $0.0010(2)$ & $-0.0095(3)$ \\
O6 & $0.0071(2)$ & $0.0090(3)$ & $0.0113(3)$ & $-0.00036(19)$ & $0.00169(19)$ & $-0.00004(19)$ \\
C1 & $0.0084(3)$ & $0.0081(3)$ & $0.0084(3)$ & $-0.0013(2)$ & $-0.0006(2)$ & $0.0017(2)$ \\
C2 & $0.0082(3)$ & $0.0089(3)$ & $0.0120(3)$ & $0.0016(2)$ & $0.0023(2)$ & $0.0013(3)$ \\
\hline
\end{tabular}

Geometric parameters $\left(\AA,{ }^{o}\right)$

\begin{tabular}{|c|c|c|c|}
\hline $\mathrm{Ni1}-\mathrm{O} 1^{\mathrm{i}}$ & $2.0302(6)$ & $\mathrm{Ni} 2-\mathrm{O} 4^{\mathrm{iv}}$ & $2.1007(7)$ \\
\hline $\mathrm{Nil}-\mathrm{O} 1^{\mathrm{ii}}$ & $2.0302(6)$ & $\mathrm{O} 1-\mathrm{C} 1$ & $1.2593(10)$ \\
\hline $\mathrm{Ni1}-\mathrm{O} 2$ & $2.0503(6)$ & $\mathrm{O} 2-\mathrm{C} 1$ & $1.2546(10)$ \\
\hline $\mathrm{Ni} 1-\mathrm{O} 2^{\mathrm{iii}}$ & $2.0504(6)$ & $\mathrm{O} 3-\mathrm{C}^{\mathrm{v}}$ & $1.2618(10)$ \\
\hline $\mathrm{Ni} 1-\mathrm{O} 3^{\mathrm{iii}}$ & $2.0942(6)$ & $\mathrm{O} 4-\mathrm{C} 2$ & $1.2607(10)$ \\
\hline $\mathrm{Ni1}-\mathrm{O} 3$ & $2.0942(6)$ & $\mathrm{O} 5-\mathrm{H} 3$ & $0.89(2)$ \\
\hline $\mathrm{Ni} 2-\mathrm{O}^{\mathrm{iv}}$ & $2.0255(7)$ & $\mathrm{O} 5-\mathrm{H} 4$ & $0.832(18)$ \\
\hline $\mathrm{Ni} 2-\mathrm{O} 5$ & $2.0256(7)$ & $\mathrm{O} 6-\mathrm{H} 5$ & 0.787 (18) \\
\hline $\mathrm{Ni} 2-\mathrm{O} 6$ & $2.0663(6)$ & O6- - H6 & 0.837 (18) \\
\hline $\mathrm{Ni} 2-\mathrm{O}^{\mathrm{iv}}$ & $2.0664(6)$ & $\mathrm{C} 1-\mathrm{H} 1$ & 0.9500 \\
\hline $\mathrm{Ni2}-\mathrm{O} 4$ & $2.1006(7)$ & $\mathrm{C} 2-\mathrm{H} 2$ & 0.9500 \\
\hline
\end{tabular}




\begin{tabular}{|c|c|c|c|}
\hline $\mathrm{O} 1^{\mathrm{i}}-\mathrm{Ni} 1-\mathrm{O} 1^{\mathrm{ii}}$ & $180.00(3)$ & $\mathrm{O} 6-\mathrm{Ni} 2-\mathrm{O} 4$ & $90.36(3)$ \\
\hline $\mathrm{O} 1 \mathrm{i}-\mathrm{Ni} 1-\mathrm{O} 2$ & $89.49(2)$ & $\mathrm{O} 6^{\mathrm{iv}}-\mathrm{Ni} 2-\mathrm{O} 4$ & $89.64(3)$ \\
\hline $\mathrm{O} 1 \mathrm{ii}-\mathrm{Ni} 1-\mathrm{O} 2$ & $90.51(2)$ & $\mathrm{O} 5^{\mathrm{iv}}-\mathrm{Ni} 2-\mathrm{O} 4^{\mathrm{iv}}$ & $90.47(3)$ \\
\hline $\mathrm{O} 1^{\mathrm{i}}-\mathrm{Ni} 1-\mathrm{O} 2^{\mathrm{iii}}$ & $90.51(2)$ & $\mathrm{O} 5-\mathrm{Ni} 2-\mathrm{O} 4^{\mathrm{iv}}$ & $89.53(3)$ \\
\hline $\mathrm{O} 1^{\mathrm{ii}}-\mathrm{Ni} 1-\mathrm{O} 2^{\mathrm{iii}}$ & $89.49(2)$ & $\mathrm{O} 6-\mathrm{Ni} 2-\mathrm{O} 4^{\mathrm{iv}}$ & $89.64(3)$ \\
\hline $\mathrm{O} 2-\mathrm{Ni} 1-\mathrm{O} 2^{\mathrm{iii}}$ & $180.00(4)$ & $\mathrm{O} 6^{\mathrm{iv}}-\mathrm{Ni} 2-\mathrm{O} 4^{\mathrm{iv}}$ & $90.36(3)$ \\
\hline $\mathrm{O} 1^{\mathrm{i}}-\mathrm{Ni} 1-\mathrm{O} 3^{\mathrm{iii}}$ & $87.42(2)$ & $\mathrm{O} 4-\mathrm{Ni} 2-\mathrm{O}^{\mathrm{iv}}$ & 180.0 \\
\hline 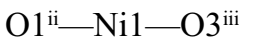 & $92.58(2)$ & $\mathrm{C} 1-\mathrm{O} 1-\mathrm{Ni} 1^{\mathrm{vi}}$ & $120.79(5)$ \\
\hline $\mathrm{O} 2-\mathrm{Ni} 1-\mathrm{O} 3^{\mathrm{iii}}$ & $93.27(2)$ & $\mathrm{C} 1-\mathrm{O} 2-\mathrm{Ni} 1$ & $125.50(6)$ \\
\hline 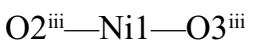 & $86.73(2)$ & $\mathrm{C} 2^{\mathrm{v}}-\mathrm{O} 3-\mathrm{Ni} 1$ & $126.31(6)$ \\
\hline $\mathrm{O} 1{ }^{\mathrm{i}}-\mathrm{Ni} 1-\mathrm{O} 3$ & $92.58(2)$ & $\mathrm{C} 2-\mathrm{O} 4-\mathrm{Ni} 2$ & $133.73(6)$ \\
\hline $\mathrm{O} 1{ }^{\mathrm{ii}}-\mathrm{Ni} 1-\mathrm{O} 3$ & $87.42(2)$ & $\mathrm{Ni} 2-\mathrm{O} 5-\mathrm{H} 3$ & $121.7(12)$ \\
\hline $\mathrm{O} 2-\mathrm{Ni} 1-\mathrm{O} 3$ & $86.73(2)$ & $\mathrm{Ni} 2-\mathrm{O} 5-\mathrm{H} 4$ & $126.1(12)$ \\
\hline $\mathrm{O} 2{ }^{\mathrm{iii}}-\mathrm{Ni} 1-\mathrm{O} 3$ & $93.27(2)$ & $\mathrm{H} 3-\mathrm{O} 5-\mathrm{H} 4$ & $110.0(16)$ \\
\hline $\mathrm{O}^{\mathrm{iii}}-\mathrm{Ni} 1-\mathrm{O} 3$ & 180.0 & $\mathrm{Ni} 2-\mathrm{O} 6-\mathrm{H} 5$ & $107.5(12)$ \\
\hline $\mathrm{O}^{\mathrm{iv}}-\mathrm{Ni} 2-\mathrm{O} 5$ & $180.00(2)$ & $\mathrm{Ni} 2-\mathrm{O} 6-\mathrm{H} 6$ & $114.6(11)$ \\
\hline $\mathrm{O}^{\mathrm{iv}}-\mathrm{Ni} 2-\mathrm{O} 6$ & $90.62(3)$ & $\mathrm{H} 5-\mathrm{O} 6-\mathrm{H} 6$ & $103.0(16)$ \\
\hline $\mathrm{O} 5-\mathrm{Ni} 2-\mathrm{O} 6$ & $89.38(3)$ & $\mathrm{O} 2-\mathrm{C} 1-\mathrm{O} 1$ & $123.70(8)$ \\
\hline $\mathrm{O} 5^{\mathrm{iv}}-\mathrm{Ni} 2-\mathrm{O}^{\mathrm{iv}}$ & $89.38(3)$ & $\mathrm{O} 2-\mathrm{C} 1-\mathrm{H} 1$ & 118.1 \\
\hline $\mathrm{O} 5-\mathrm{Ni} 2-\mathrm{O}^{\mathrm{iv}}$ & $90.62(3)$ & $\mathrm{O} 1-\mathrm{C} 1-\mathrm{H} 1$ & 118.1 \\
\hline $\mathrm{O} 6-\mathrm{Ni} 2-\mathrm{O}^{\mathrm{iv}}$ & 180.0 & $\mathrm{O} 4-\mathrm{C} 2-\mathrm{O} 3^{\mathrm{vii}}$ & $125.14(8)$ \\
\hline $\mathrm{O}^{\mathrm{iv}}-\mathrm{Ni} 2-\mathrm{O} 4$ & $89.53(3)$ & $\mathrm{O} 4-\mathrm{C} 2-\mathrm{H} 2$ & 117.4 \\
\hline $\mathrm{O} 5-\mathrm{Ni} 2-\mathrm{O} 4$ & $90.47(3)$ & $\mathrm{O}^{\mathrm{vii}-\mathrm{C} 2-\mathrm{H} 2}$ & 117.4 \\
\hline
\end{tabular}

Symmetry codes: (i) $x,-y+5 / 2, z+1 / 2$; (ii) $-x+1, y-1 / 2,-z+1 / 2$; (iii) $-x+1,-y+2,-z+1$; (iv) $-x,-y+2,-z$; (v) $x,-y+3 / 2, z+1 / 2$; (vi) $-x+1, y+1 / 2,-z+1 / 2$; (vii) $x,-y+3 / 2, z-1 / 2$.

Hydrogen-bond geometry $\left(A,{ }^{\circ}\right)$

\begin{tabular}{lllll}
\hline$D-\mathrm{H} \cdots A$ & $D-\mathrm{H}$ & $\mathrm{H} \cdots A$ & $D \cdots A$ & $D-\mathrm{H} \cdots A$ \\
\hline $\mathrm{O} 6-\mathrm{H} 5 \cdots \mathrm{O} 3^{\text {viii }}$ & $0.787(18)$ & $1.985(18)$ & $2.7312(9)$ & $158.1(16)$ \\
$\mathrm{O} 5-\mathrm{H} 3 \cdots \mathrm{O} 2$ & $0.89(2)$ & $1.87(2)$ & $2.7522(9)$ & $171.2(19)$ \\
$\mathrm{O} 5-\mathrm{H} 4 \cdots 4^{\text {viii }}$ & $0.832(18)$ & $1.898(18)$ & $2.7271(10)$ & $174.1(17)$ \\
$\mathrm{O} 6-\mathrm{H} 6 \cdots 1^{\text {iv }}$ & $0.837(18)$ & $1.926(18)$ & $2.7610(9)$ & $175.5(16)$ \\
\hline
\end{tabular}

Symmetry codes: (iv) $-x,-y+2,-z$; (viii) $-x, y+1 / 2,-z+1 / 2$. 